\title{
INCREASING FUNCTIONALITY AND THE QUALITY OF LIFE THROUGH MEDICAL REHABILITATION IN PATIENTS WITH PARKINSON'S DISEASE
}

\author{
Moldovan Andreea Alexandra², Dogaru Gabriela ${ }^{1,2}$ \\ 1University of Medecine and Pharmacy "Iuliu Hatieganu” Cluj \\ ${ }^{2}$ Rehabilitation Hospital Cluj
}

\begin{abstract}
Parkinson's disease is a neurodegenerative disorder of the extrapyramidal nervous system, due to the destruction of pigmented dopaminergic neurons, particularly in the substantia nigra of the brainstem. The age range at which this symptomatology most frequently occurs is between 50 and 70 years. In Romania, incidence is 4000-5000 new cases per year, with a prevalence of 30,00050,000 cases for the whole country. There are three major clinical symptoms: rest tremor, extrapyramidal rigidity, akinesia. Physical-kinetic treatment is extremely important, helping patients to maintain their professional and extraprofessional activities for as long as possible, improving walking disorders, hypokinesia and the flexion tendency, while being supported by adequate psychotherapy. The work techniques should be chosen depending on the clinical form and the severity of the functional deficit, as well as on the individual peculiarities of each patient.The whole medical rehabilitation program should be carried out in a peaceful, relaxing environment, taking into consideration the quality not the quantity of the exercises, while a decisive role is played by the family, which must continuously encourage and support the patient.
\end{abstract}

Key Words: Parkinson's disease, medical rehabilitation, occupational therapy, kinetotherapy 


\section{Introduction}

The history of Parkinson's disease began in 1817, when James Parkinson described among motor disorders a disease termed "shaking palsy", which was later named after him.

This is a neurodegenerative disease of the extrapyramidal nervous system, due to the destruction of pigmented dopaminergic neurons, particularly in the substantia nigra of the brainstem. This degeneration leads to a dopamine deficiency in the striatal projection areas of these neurons. In 1912, the presence of characteristic hyaline inclusions, which were subsequently termed Lewy bodies, was also found.

The age range at which the symptomatology of Parkinson's disease most frequently occurs is between 50 and 70 years. These symptoms become manifest when $70-80 \%$ of striatal dopaminergic neurons and $50 \%$ of nigral dopaminergic neurons are lost.

The disease frequency is considerable, with a 1:40,000 prevalence in the general population, while in the population aged over 40 years, prevalence is 1:200, with a predominance in the male sex, $1.25: 1$; only $5-15 \%$ of the cases are accounted for by heredity.

In Romania, incidence is 4000-5000 new cases per year, with a prevalence of 30,000-50,000 cases for the whole country.

Although idiopathic Parkinson's disease is usually sporadic, the genetic component of the disease has also been discussed. A major finding of recent years is the mapping and cloning of several genes that cause the single gene transmission forms of the disease. However, these seem responsible for the disease in a relatively small number of families, and the genetic substrate of the majority of the cases has no clear pattern [1].

The role of environmental factors in the etiology of Parkinson's disease is not certain, but in the course of time, correlations between the disease and several factors such as steel industry, agriculture, pesticide exposure, paper and wood industry have been established. The repeated exposure to an environmental toxin might explain the age range at which the disease occurs [4].

Studies performed on monozygotic and dizygotic twins have suggested that the disease rarely has a genetic basis. Positron emission tomography has shown that asymptomatic twins frequently have striatal dopamine uptake anomalies.

The spread of encephalitis lethargica (sleep disease) throughout the world in the 1920 's led to parkinsonism in $80 \%$ of these patients. However, there is a difference between postencephalic parkinsonism and Parkinson's disease; the subjects of the first category do not have Lewy bodies in the substantia nigra. Other studies have failed to prove an association between a virus and Parkinson's disease [2, 3].

During dopamine oxidation, toxic free oxygen radicals are produced. Oxidative stress leads to increased iron concentrations, low glutathione and peroxidase levels, while the activity of MAO-B (a dopamine mediator) tends to increase with age.

Cranial trauma in the pathogenesis of the disease is debatable, and so is the location of newly formed lesions in the structures responsible for the development of Parkinson's syndrome. It has been demonstrated that vascular factors such as arterial hypertension can favor Parkinson's disease [4].

Parkinson's disease is dominated by three major symptoms: rest tremor, extrapyramidal rigidity, akinesia.

Starting from the hypothesis that medical rehabilitation treatment is indispensable to the therapeutic program in Parkinson's disease, its effectiveness was studied in order to increase the functionality and the quality of life of patients with Parkinson's disease.

Physical-kinetic treatment is extremely important, helping patients to maintain their 
professional and extraprofessional activities for as long as possible, improving walking disorders, hypokinesia and the flexion tendency, while being supported by adequate psychotherapy. The work techniques should be chosen depending on the clinical form and the severity of functional deficit, as well as on the individual peculiarities of each patient. Treatment should be initiated since the onset of the disease, and in the fully established disease, it will be related to the effectiveness of drug therapy.

In the "on" period, therapy is limited to increasing the speed of movement execution and coordination, while in the "off" period, the patient must attend a functional program, being incapable of exercise. The participation in the rehabilitation program is determined by the patient's confidence in the therapist.

The entire program should be carried out in a peaceful, relaxing environment, taking into consideration the quality, not the quantity of exercises, while a decisive role is played by the family, which should continuously understand, encourage and support the patient $[5,6]$.

\section{Material and method}

The study was performed in the Clinical Rehabilitation Hospital ClujNapoca, in the Clinical Rehabilitation Hospital Băile Felix, as well as in the Clinical Emergency Hospital Cluj-Napoca, Department of Neurology, in the period April-May 2014.

The study included 20 patients with Parkinson's disease, of which 14 men and 6 women, patients with stages 1,2 and 3 of the disease. Ten of these received anti-Parkinson drug therapy (group B), and the other 10 received in addition to drug therapy, individualized rehabilitation treatment for two weeks (group A).

The rehabilitation program consisted of kinetotherapy (active mobilizations, recovery of initiative and walking speed, recovery of grip, respiratory gymnastics, facial expression exercises, exercises for pelvic muscle toning, recovery of transfers, recovery of balance), occupational therapy, medical massage, magnetodiaflux, medium frequency interferential currents.

The Barthel scale was used for the evaluation of 10 items: walking, transfer, climbing stairs, feeding, hygiene, dressing, toilet use, continence, micturitions, bathroom use before and after medical rehabilitation treatment.

The results were graphically recorded, based on analytical tables. The data obtained were processed in Microsoft Excel, using the Student test for paired samples.

We used the Barthel index to measure the degree of independence and performance in activities of daily life, with the aim of increasing the quality of life of patients, their functionality and independence.

The exclusion criteria of this study were contraindications of physiokinetotherapy due to associated pathology or refusal of the patient.

\section{Results and discussion}

\section{Analysis of the groups depending on sex distribution}

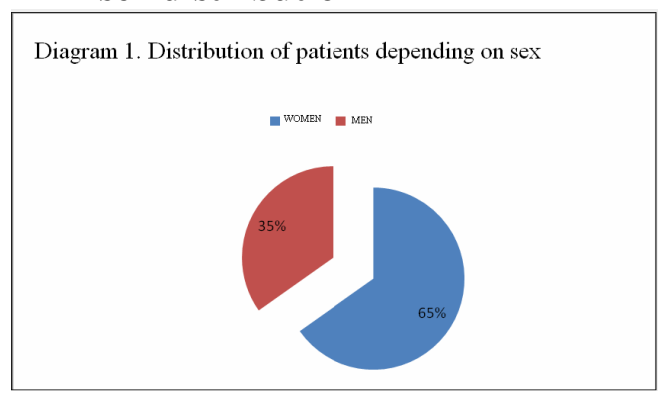

This study shows that Parkinson's disease was more frequent in the male sex, in a proportion of $65 \%$, which is in accordance with the literature data.

\section{Analysis of the groups depending on age decades}

In the evaluated groups, the age limits ranged between 50 and 81 years, with a mean age of 60 years. 
Diagram 2. Distribution by age groups

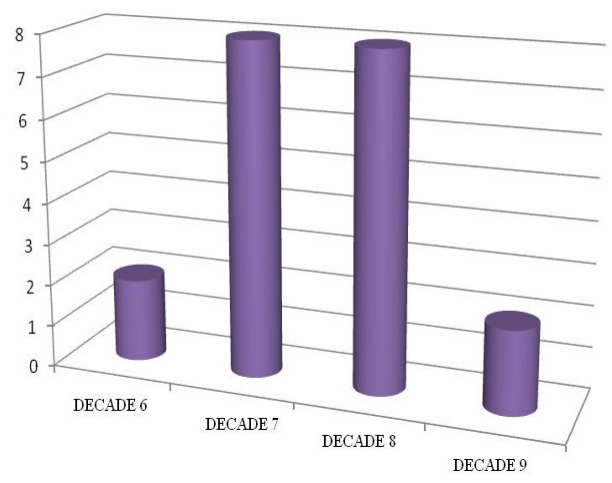

Diagram 2 shows the distribution of all studied patients depending on age, in order to monitor the disease frequency from this point of view. The highest incidence of Parkinson's disease was found for decades 7 and 8, i.e. between 60 and 79 years, while there were two patients $(10 \%)$ in decade 6 and another two patients in decade 9 .

\section{Analysis of the groups depending on the patient's occupation}

Of all patients, $2(10 \%)$ continued to be active, and the rest of 18 , i.e. $90 \%$ of them were retired due to advanced age and to the disease that limited effective work. The pursued aim is to help patients with working potential to maintain their professional activities for as long as possible.

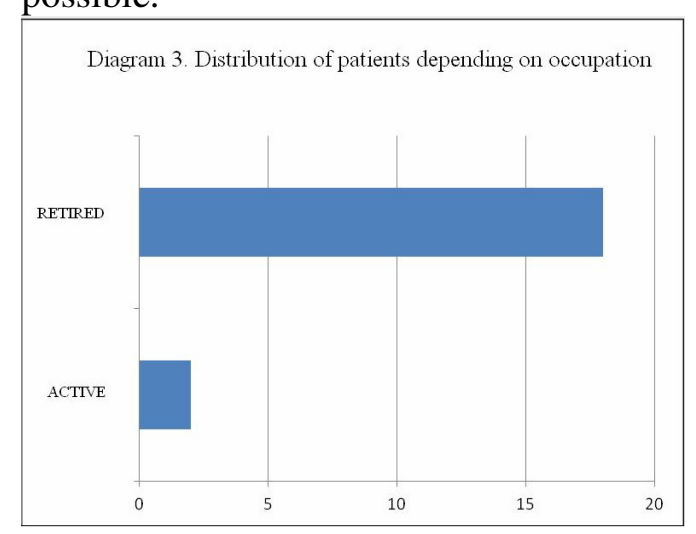

\section{Graphical representation depending on the onset symptom of the disease}

Following analysis, it was found that the predominant onset symptom was tremor in 11 patients (55\%), while rigidity accompanied by muscle pain was seen in 6 patients (30\%), and bradykinesia in 3 patients (15\%) [Diagram 4].

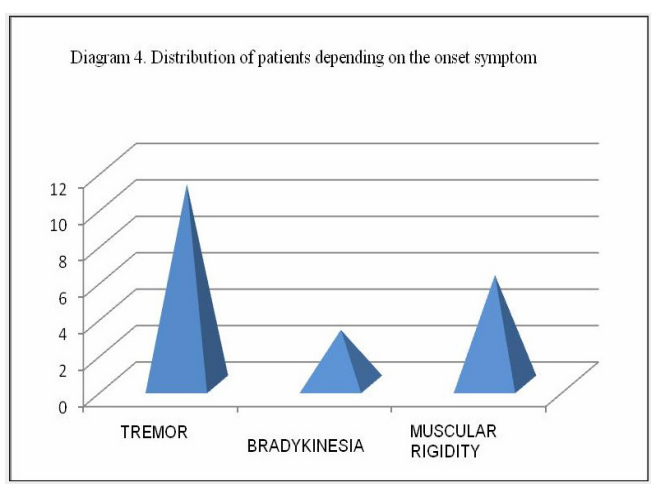

\section{Graphical representation depending on the improvement of walking} Diagram 5. Distribution of patients according to their
degree of dependence for walking at admission

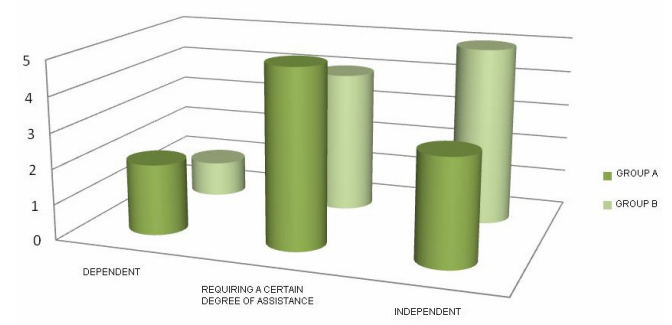

In order to calculate the degree of walking independence at admission, we used the Barthel scale. We concluded that of the 20 patients, only $8(40 \%)$ were completely independent, $9(45 \%)$ required a certain degree of assistance for walking, and 3 (15\%) were completely dependent on their entourage [Diagram 5].

The aim of walking reeducation was to improve balance, the patients' ability to walk alone, and to diminish the risk of fall, in order to increase functionality and the quality of life of these patients. 


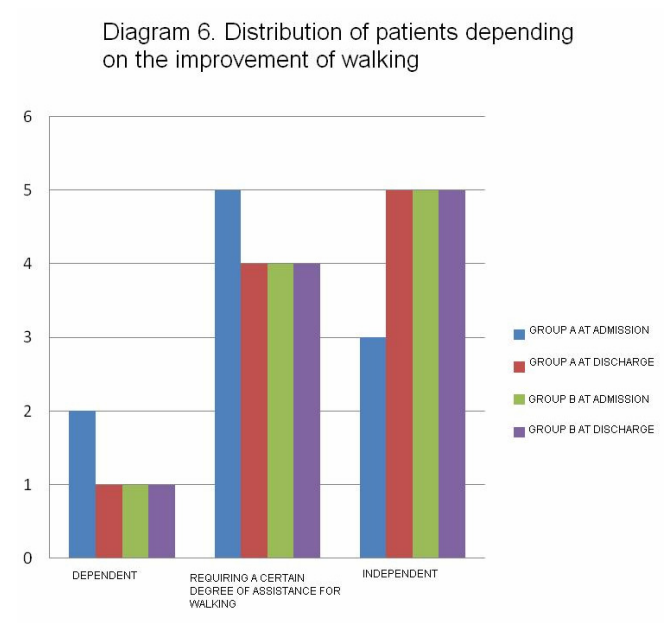

The analysis of the diagram shows that patients of group A, who attended a rehabilitation program, had an improvement in walking, while patients of group B had no improvement. Statistically, $20 \%$ of group A patients were initially dependent, 50\% required a certain degree of assistance, and $30 \%$ were independent; after discharge, the percentage of dependent patients decreased to $10 \%$, the proportion of patients requiring a certain degree of assistance decreased to $40 \%$, and the number of independent patients increased to $50 \%$. It can be seen that after drug treatment, the percentages of all patient categories in group B were unchanged [Diagram 6].

\section{Graphical representation depending on the improvement of feeding \\ Diagram 7. Distribution of patients depending on the improvement of feeding}

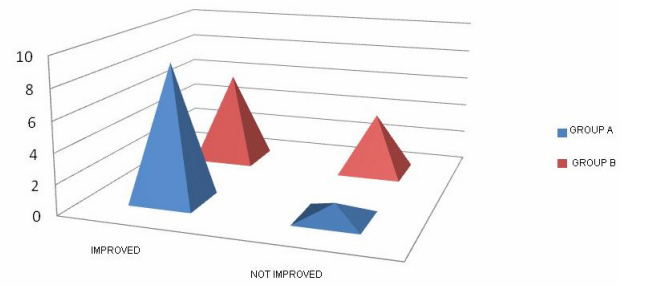

The analysis of Diagram 7 clearly shows that patients of the study group A had a greater number of improvements regarding feeding and the degree of dependence related to it. In group A, $90 \%$ of patients had improvements, while in group $\mathrm{B}$, the group of patients who received drug therapy, only $60 \%$ of patients had improvements, the rest of $40 \%$ having the same difficulties regarding feeding.

\section{Distribution of patients depending on the improvement of micturition and incontinence}

Patients of this study had sphincter incontinence and imperative micturition before treatment.
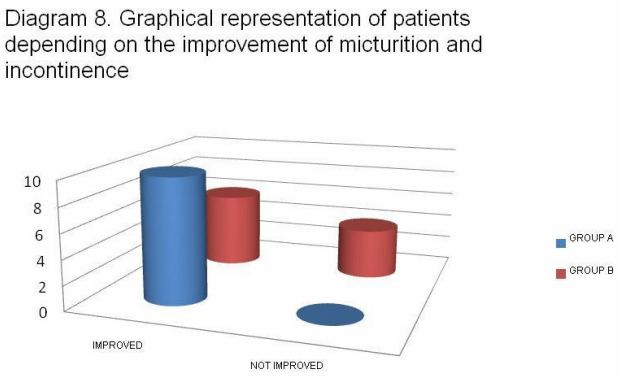

Based on Diagram 8, it can be concluded that all patients of group A had a certain improvement of incontinence or laborious micturition after treatment. In group B, patients who received drug therapy alone had fewer improvements after day 10 of treatment. Statistically, only $60 \%$ of patients in group B had improvements, the rest of $40 \%$ of group B patients showing no improvement, while $100 \%$ of group A patients had improvements.

\section{Distribution of patients depending on their degree of dependence in carrying out activities of daily life using the Barthel index}

Diagram 9. Distribution of patients depending on their total degree of dependence according to the Barthel index

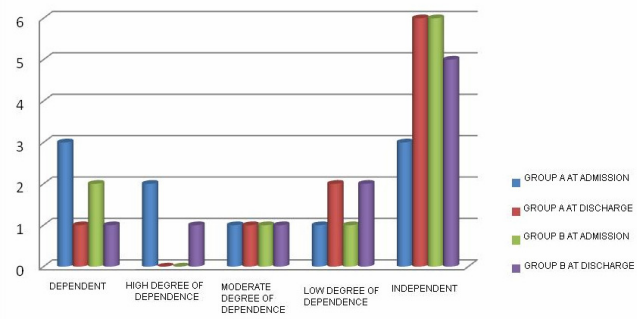


The analysis of Diagram 9 evidences small improvements in the degree of dependence of group B patients, according to the Barthel scale, but significant improvements can be seen in group A patients, who received rehabilitation treatment in addition to drug therapy. The benefits of physiokinetotherapy in reducing the degree of dependence in patients with Parkinson's disease are obvious.

From a statistical point of view, $80 \%$ of patients of group A had improvements in functionality and implicitly, in the quality of life, while only $40 \%$ of patients of group B had improvements.

\section{CONCLUSIONS}

The study group, including patients who attended a physical-kinetic program in addition to drug treatment, had $80 \%$ increases in functionality and implicitly, in the quality of life, while in the control group, only half of these improvements were found after drug therapy alone.

Kinetotherapy considerably improved walking, reducing the degree of dependence in patients of the study group.

Occupational therapy significantly contributed to increasing the patients' performance in carrying out activities of daily life.

The use of individualized physicalkinetic techniques for each patient allowed to improve their condition.

Patients who attended a rehabilitation program know maintenance exercises that they will be able to perform at home for at least 30 minutes every day, and their families know the details of how to make the patient's life easier.

\section{Bibliography}

1. http://educatie-fizicakineto/pdf/studenti/cursuri\%master/note curs_nervos.pdf

2. Lăcrimioara Perju-Dumbravă. Strategii terapeutice moderne în Boala Parkinson. Cluj-Napoca: Editura Medicală Universitară "Iuliu Ha $\square$ ieganu"; 2002. p. 29- 79.

3. Marilena Kory-Mercea, $\square$ tefania Kory Calomfirescu. Kinetoterapia pacien $\square$ ilor cu Boală Parkinson. Cluj-Napoca: Editura Risoprint; 2009.

4. www.uni.edu/walsh/balsalganglia-2.ipg

5. Roger C. Duvoisin, M.D. Parkinson's disease. A guide for Patient and Family. 3th ed. New York: Raven Press; 1991.Schapira A.H.W. Progress in Parkinson's disease. New York: Raven Press; 2003. 